\title{
EVALUATION OF SENSOR SIGNAL PROCESSING METHODS IN TERMS OF INFORMATION THEORY
}

\author{
PATRIK FlegneR*, JÁn KaČUR \\ Institute of Control and Informatization of Production Processes, Faculty BERG, Technical University of \\ Košice, Boženy Němcovej 3, 04001 Košice, Slovak Republic \\ * corresponding author: patrik.flegner@tuke.sk
}

\begin{abstract}
The paper deals with the examination of basic methods of evaluation of sensor signals in terms of the information content of the given method and the used technical means. In this respect, methods based on classical analog systems, digital systems in the time domain of signal processing, hybrid systems and digital systems evaluating signal in the frequency domain are compared. A significant increase in entropy in individual systems is demonstrated in the case of a more complex signal evaluation. For each measuring system, the experimental setups, results, and discussions are described in the paper. The issue described in the article is particularly topical in connection with the development of modern technologies used in the processes and subsequent use of information. The main purpose of the article is to show that the information content of the signal is increased because the signal is more complexly processed.
\end{abstract}

KEYWORDS: entropy, information, analog and digital system, spectrum, spectrogram.

\section{INTRODUCTION}

The term data mining is used today mainly in management and marketing, where it is understood as the process of obtaining information from the available data. In this "mining", various methods and procedures are used by employing among other things, modern information technologies. We no longer meet this concept in the field of control of production and technological processes. It is in this area that information as a basis for decision-making in the choice of appropriate intervention in the process, is of a fundamental importance. With the increasing complexity of processes such as controlled objects, with an increasing computing and communication technology, and progress in science disciplines, such as control theory and artificial intelligence, the classical exact control methods and new or modern methods are offered. These methods are based on the acquisition of qualitatively new types of information about the controlled and monitored process.

We can define the sketched problem of "data mining" from signal sensors in terms of information theory and signal theory.

Information, from the viewpoint of information theory eliminates uncertainty (i.e., entropy). The measure of information is the increment of probability after receiving the message.

If we accept the $A$ information that we can expect with the probability $p(A)$, then we receiv the amount of information (bit) in the sense of Shannon's entropy theorem [1]:

$$
I(A)=-\log _{2} p(A) \quad(\text { bit }) .
$$

If we quantify the information according to Shan-

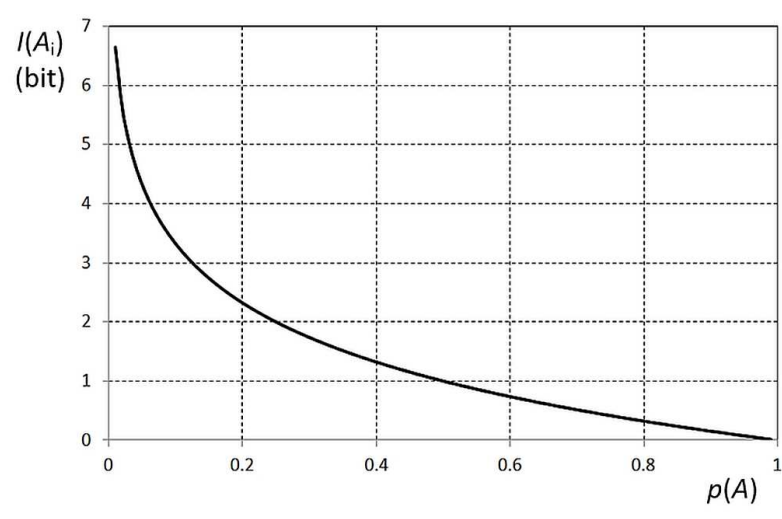

FiguRE 1. Relationship between the probability of information and its entropy.

non's theorem [2, 3] then it is valid that:

$$
\begin{aligned}
p\left(A_{i}\right)=0.5 & \Rightarrow I\left(A_{i}\right)=1 \quad \text { (bit) } \\
p\left(A_{i}\right) \rightarrow 1 & \Rightarrow I\left(A_{i}\right) \rightarrow 0 \quad \text { (bit), } \\
p\left(A_{i}\right) \rightarrow 0 & \Rightarrow I\left(A_{i}\right) \rightarrow \infty \quad \text { (bit). }
\end{aligned}
$$

From (1) and from Figure 1, it is clear that if the specific information is less probable and if this information occurs and we accept this information [4, we obtain a larger amount of information [5, 6].

The theory of information for the purposes of active and by time term periodically process of information receiving defines the information source [7]. With some simplification, based on the information theory and probability theory, we can define the information source as a probabilistic space [8]. We can write this space in mathematical formalism as follows:

$$
\varphi=\left(X^{*}, P\right),
$$


where $X$ is the final set of elements $X=$ $\left\{x_{1}, x_{2}, \ldots, x_{n}\right\}$, which we call the source alphabet and its element by the letter of the source, $X^{*}$ is the set of all the final sequences of the elements of $X$ and represents a set of possible source messages, $P$ is a probability function defined on a set $X^{*}$. Function $P$ has these properties: $P=\left\{p\left(x_{i}\right) ; x_{i} \in X\right\} \in\langle 0,1\rangle$.

Obviously, the longer the fed information, the more information can be sent. Therefore, as an information content of an information source (entropy of an information source), the average entropy of the source per information is used $x$ (probability average) [9]. For stationary and ergodic sources of information, we then obtain:

$$
H(\varphi)=-\sum_{x \in X^{*}} P(x) \log _{2} P(x) \quad \text { (bit) }
$$

By analyzing the relation (3), it is possible to come to a serious conclusion, the higher the information content of the source, the higher the amount of information, which is generated with a uniform probability. That is, the size of the probability space (i.e., the number of elements of set $X^{*}$ ) directly determines the "content" of a specific information source [10, 11.

From a functional point of view, we can divide the information acquisition process into several basic functions. It is clear that the key role in terms of the adequacy of the information obtained and in terms of its quantity is played by the sensor during the measurement [12, 13].

Other processes can only damage the acquired information or destroy it altogether. Consequently, it is not possible to add relevant information to the measured variable through the processes [14. The problem lies in how to "data mine" and then "use" the maximum information contained in the signal from the sensing element.

Thus, the output analog signal of the sensor in operation can be understood as the bearer of the information, as a continuous information source. It is demonstrated in the literature [15, 16 that the maximum amount of information is contained in such sensor signal having a limited average power $\mathrm{P}_{m}$ and whose amplitude probability density distribution $p(x)$ is by Gaussian distribution on interval $x \in\left\langle x_{\min }, x_{\max }\right\rangle$ :

$$
p(x)=\frac{1}{\sqrt{2 \pi e \mathrm{P}_{m}}} \exp -\frac{x^{2}}{2 \mathrm{P}_{m}} .
$$

Its information content (4) then acquires the maximum value:

$$
\max H_{a}=\frac{1}{2} \log _{2}\left(2 \pi e \mathrm{P}_{m}\right) \quad \text { (bit)., }
$$

where $e$ is the operator with an expected value. Information content by (5) is only the theoretical value because it assumes the ability of the sensor to generate at its output infinitely many amplitude levels of the signal from the interval $x \in\left\langle x_{\min }, x_{\max }\right\rangle$. With a real sensor, this is not possible due to its limited sensitivity and its inaccuracy.

The sensor with the accuracy class $\delta$ can generate a signal of about $n=\frac{1}{2 \delta}+1$ amplitude levels. This then causes a decrease of the information content of the sensor towards the theoretical value (5) and in accordance with (3).

Another important moment that essentially decides about "data mining" is that the maximum amount of information contained in the analog signal of the sensor is the signal evaluation process itself. At present we can talk about two basic ways:

- evaluation of the amplitude of the analog signal in the time domain by a standard analog or modern digital system;

- evaluating the amplitude of the analog signal in the frequency domain using a digital measurement system.

As mentioned above, from (3) follows that the sensor as a discrete information source has the higher information content, the more amplitude levels of its output signal $x(t)$ we can distinguish.

With certain simplifications, when we neglect the sensitivity and accuracy class of the real sensor, we can deduce from the entropy $H_{a}$ equation (4) of the analog signal that is continuous both in time and amplitude on the final amplitude range.

\section{AnAlog MeAsuring System}

The measurement system generally represents a summary of the elements that provide the measurement task. The behaviour of the measured signal is interpreted mainly by using the signal analysis at certain points of amplitude, time and the frequency view. From these characteristics, it is possible to obtain information about a process that could not be captured using the basic signal processing functions. This includes the processing of average data values, determination of their distribution, correlations, transformations, and also the functions necessary to describe deterministic or stochastic signals in static processes or in transition processes [17. Signal analyses are most often solved by an external host computer without requiring a real-time operation [18]. As an example, the determination of the sampling period of a process variable based on the analysis of the frequency spectrum of the measured signal according to Shannon theorem can be used [19].

The processing of this signal in the time domain deals about analysis of its overall amplitude. In the past and in many cases even today, this is the most common way of evaluating the measurement of physical variables [20]. The visual display of the corresponding amplitude of the one-way signal of the sensor is realized by means of an analog apparatus calibrated in the corresponding physical units (see Figure 2).

As mentioned above, analog measurement systems are classified based on the accuracy class $\delta(\%)$ e.g., 


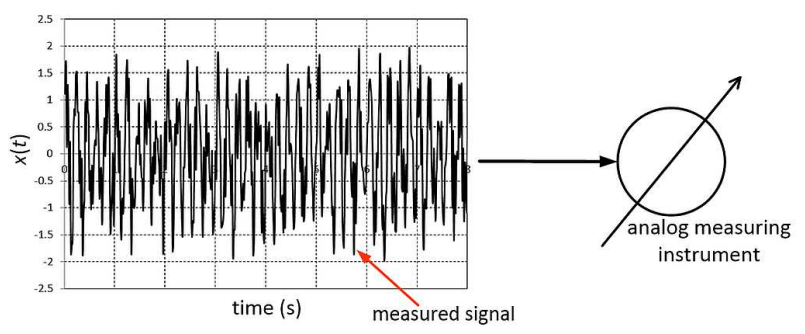

Figure 2. Signal evaluation by analog measuring instrument.

$0.01,0.02,0.05,1.0,1.5$ and 2.5 . For the accuracy class, it is valid $\delta= \pm \frac{\max \varepsilon}{\text { range }} 100 \%$. It causes the socalled uncertainty band of a relative width $\varepsilon=2 \delta$ near the result of a measurement. An analog measuring system with a relative error $\delta$ provides $n$ of the distinguishable amplitudes of the measured physical variable, with regards to the following equation:

$$
n=\frac{1}{2 \delta}+1=\frac{1}{\varepsilon}+1 \text {. }
$$

If, for simplicity, we assume an uniform distribution of the probability density of the measured quantity, i.e., all values have the same probability of occurrence $p=\frac{1}{n}$, it is possible to simplify the differential entropy of an analog measuring system with a given accuracy class $\delta$ based on (3) into the following form:

$$
H_{a \delta}=\log _{2} n=\log _{2}\left(\frac{1}{2 \delta}+1\right) \quad \text { (bit) }
$$

This equation gives the maximum boundary value of information that one measurement can contain. If, for example, the relative error of the analog measuring system $\delta=0.01 \%$, it allows the instrument to measure 51 different measured values on a given range. Then, according to (7), we receive the information content of this measurement system $H_{a \delta}=\log _{2} 51=5.67$ (bit).

\section{Digital MEASURING SYSTEM}

Nowadays, in the practical applications of the theory of the automatic control or digital signal processing [21, we very often counter the issue of communication of discrete technical devices with a continuous environment 22 . The bridges, which enable us to connect digital and continuous worlds, are digital-toanalog (DAC) and analog-to-digital (ADC) converters. At present, the evaluation of a measurement of the variable by digital systems prevails.

Digital measurement systems are based on the digitization 23 of the analog signal by the $m$-bit analogto-digital converter. If the width of the $\mathrm{AD}$ converter is $m$-bits, then this converter will distinguish, on the interval of $x \in\left\langle x_{\min }, x_{\max }\right\rangle$, the total $n=2^{m}$ amplitude signal levels. The differential entropy of this sampled signal is generally given by (3). In the case of a uniform distribution of signal probability, the simplified equation applies:

$$
H_{\mathrm{DIG} m}=\log _{2} n=\log _{2} 2^{m}=m \quad \text { (bit). }
$$

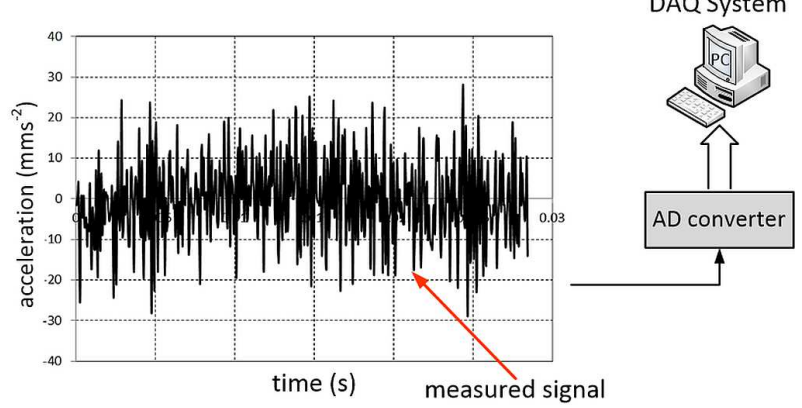

Figure 3. Digital signal evaluation by digital system.

If, for example, we consider a common 12-bit AD converter in technical practice, this allows us to distinguish up to the given signal range of $n=2^{12}=4096$ different levels i.e., measured values. Assuming the uniform distribution of the probability of the measured values, we receive the information content of this measuring system $H_{\mathrm{DIG} 12}=\log _{2} 4096=12$ (bit).

From the comparison results that in practice are valid $H_{a \delta}<H_{\mathrm{DIGm}}$. Thus, numerical methods achieve significantly higher accuracy than analog methods. They have better static properties, but the price is worse dynamic properties. An illustrative diagram of a signal evaluation by a digital system is shown in Figure 3

\section{HYBRID MEASURING SYSTEM}

Another type of digital measurement systems are systems based not on the processing of the sampled sensor signal but on the evaluation of the analog signal of the sensor itself. The analog signal of the sensor is evaluated by a special set of analog and digital circuits. This is a hybrid measurement system, although its fundamental is the use of special programmable digital circuits. To calculate the differential entropy of such measuring systems, we usually have to approach them individually.

As an example of a digital or hybrid measuring system, it is possible to include a device for measuring a Young's elastic modulus of steel ropes [24.

This is a method of indirectly measuring the elasticity modulus of steel rope under traction based on the measurement of propagation velocity of longitudinal wave caused by a mechanical shock. From a physical point of view, the method relies on a known dependence between the rate of sound propagation in the material $v\left(\mathrm{~ms}^{-1}\right)$ and modulus of elasticity $E$ $(\mathrm{MPa})$ of steel rope, whose mass density of material is $\rho\left(\mathrm{kg} \mathrm{m}^{-3}\right)$ [25, 26]. For a more accurate idea of dependence, we also present the following equation:

$$
E=v^{2} \rho \quad(\mathrm{MPa})
$$

The velocity of the propagation of the longitudinal acoustic wave in the steel rope can be converted to two time-shifted $\tau$ pulses using suitable sensors and pre-amplifiers [27. By a time shift $\tau$, the time 


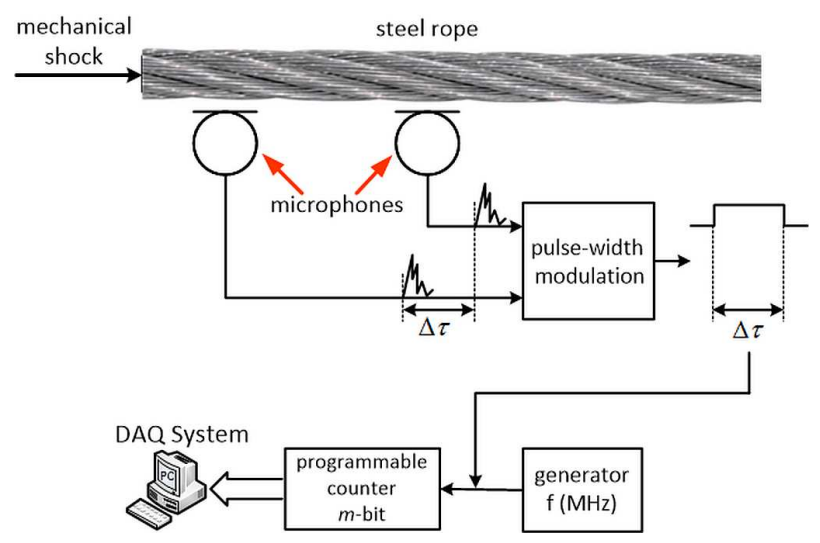

FigURE 4. Hybrid measurement system with a pulse width modulation signal.

period after which the mechanical shock from one cross-section of the rope passes to the other is meant. The implemented flip-flop circuit converts these two time-shifted pulses into one width-modulated pulse. The counter is controlled by the impulse so that it only works for the duration $\tau$ commensurable to the velocity of the wave propagation velocity and also to indirectly measured modulus of elasticity of a steel rope (see Figure 4). The presented hybrid measuring system is implemented in practice and is still functional for the purpose of assessing the quality and damage of the steel rope 28 .

Counting the generated pulses by the counter for a time $\tau$ may cause an inaccuracy of a unit size. This means that in one measurement we obtain the uncertainty of $\varepsilon=\left(\tau f_{G}\right)^{-1}$ for the impulses $\tau f_{G}$. The maximum distinguishable number of levels $n$ of measured variable is:

$$
n=\frac{1}{\varepsilon}+1=\tau f_{G}+1,
$$

where the value of one represents a zero amplitude value.

This basic equation 10 is valid for a digital measurement and shows that an increase in the number of distinguishable levels and thus the entropy of the measurement will be achieved by increasing the clock frequency $f_{G}$ of the generator. It is assumed that this frequency $f_{G}$ of the used generator is determined without an error. If, for example, we use the generator with a frequency $f_{G}=10(\mathrm{MHz})$, then on a unit scale $\tau \in\langle 0,1\rangle$ in seconds, we can distinguish $n=10^{7}$ levels, which correspond to an entropy $H_{\mathrm{HYB}}=\log _{2} 10^{7}=23.25$ (bit).

\section{Processing the Signal in the FREQUENCY DOMAIN}

The basic method of a signal processing in the frequency domain is the analysis of its spectrum (see Figure 5). It is based on the fact that the sequence of $N$ samples (i.e., the record $x_{s}$ ) of any real signal can be expressed in terms of the approximation of the sums of the unique series of $N$ harmonic components, each of which has its complex amplitude $F_{k}$, frequency $f_{k}$ and phase shift $\varphi_{k}, k=0,1,2, \ldots, N-1$, is valid equation:

$$
x_{s}(t)=\sum_{k=0}^{N-1} F_{k} e^{i k 2 \pi f_{1} t+\varphi_{k}}, \quad t \in\langle 0, T\rangle .
$$

Equation (11) is valid for the real signal that is limited by the highest frequency component $f_{s} / 2$, while we assume its periodicity with the base time period $N / f_{s}$. For the first frequency component in the spectrum (the so-called base frequency) $f_{1}$ and for the frequency resolution $\Delta f$ in the spectrum, it is valid $f_{1}=\Delta f=\frac{1}{T}=\frac{f_{s}}{N}$, where $T$ is the length of the record of analysed sensor signal in time units. The record length $T$ depends on the number of samples $N$ and the sampling frequency of signal $T=\frac{N}{f_{s}}$. In equation (11), the coefficient was limited to the range 0 to $N-1$, because in the sense of the discrete Fourier transform (DFT), the number of the spectrum lines must correspond the number of samples in the record. The spectrum is complex, thus comprised of the amplitude spectrum and the phase spectrum. The number of spectral lines represented in the spectrum is equal to the number of $N$ samples in the analysed signal recording. Due to the aliasing and the symmetry of the discrete spectrum around the axis $f_{s}$, the usable part of the complex spectrum is only until the Nyquist frequency $f_{s} / 2$. Therefore, for the frequency analysis and industrial practice, the usable number of discrete complex spectrum lines is according to 111) $N / 2$ (see Figure 6). To assess the amount of information contained in the signal spectrum, we must build on the number $n_{|F|}$ of possible shapes of amplitude spectrum and also on the number $n_{\varphi}$ of phase spectra. Due to the discreet signal evaluation, this is the final count. For simplicity, consider only the amplitude spectrum analysis, which is more common in practice. When calculating the number of possible amplitude signal spectra, we must realize that this spectrum consists of $N / 2$ spectral lines, each of which can have one of $2^{m}$ values. From a combinatorial point of view, there are variations of $N / 2$ class from $2^{m}$ elements with the repeating. Each of the amplitude levels can occur across multiple spectral lines. Then it is valid that:

$$
n_{|F|}=V_{N / 2}^{\prime}\left(2^{m}\right)=\left(2^{m}\right)^{N / 2} .
$$

Then the entropy $H_{f}$ (bit) of the measurement based on the amplitude spectrum examination of the sensor signal is given by:

$$
\begin{aligned}
H_{f}=\log _{2} n_{|F|}= & \log _{2}\left(2^{m}\right)^{N / 2} \\
& =\frac{N}{2} \log _{2} 2^{m}=\frac{N}{2} m
\end{aligned}
$$

If, for example, we used an $m=12$ (bit) sensor and an $\mathrm{AD}$ converter to digitize the analog signal, and we would evaluate the two-sided complex amplitude 


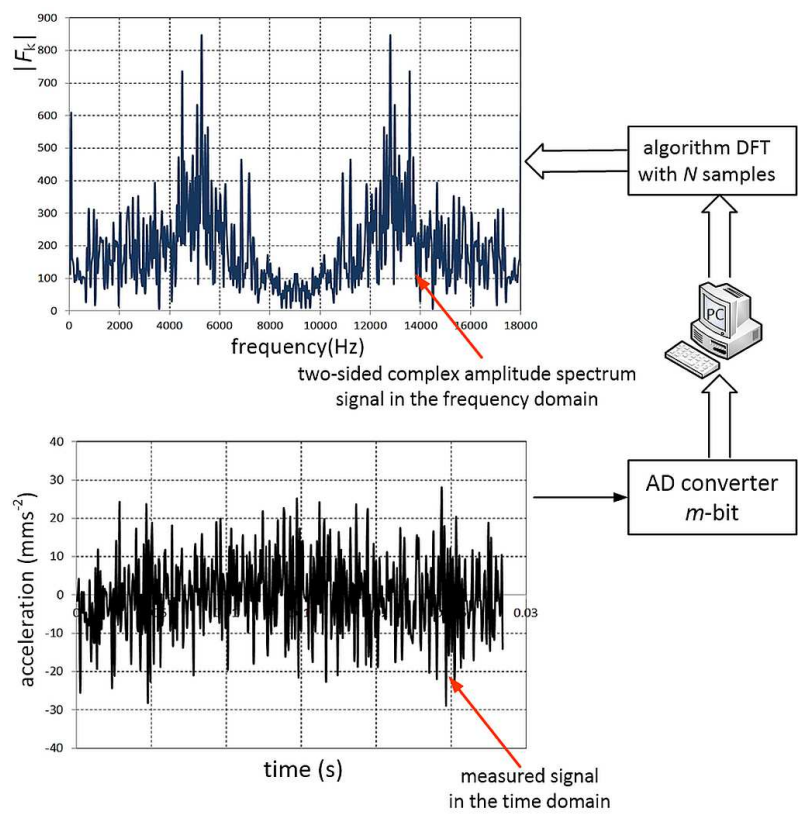

Figure 5. Signal evaluation by a digital system in the frequency domain.

spectrum of this signal with the length of $N=1024$, then the entropy of such measurement would be $H_{f}=$ $\frac{1024}{2} 12=6144$ (bit).

In Figure 6, as an example from practice, a one two-sided complex amplitude spectrum of the accompanying acoustic signal generated in disintegration of the rock by the rotation drilling is exemplified is shown [29]. The entropy of the spectrum has a value of 6144 (bit). The measurement was carried out on the horizontal laboratory drilling stand. A record of $N=1024$ samples obtained at a sampling frequency of $f_{s}=18(\mathrm{kHz})$ from the microphone signal was evaluated using a $m=12$ (bit) AD converter. The purpose of analysing this acoustic signal is to find the information in the signal that can be used for an optimal control of the drilling process [30. The basic criteria for optimizing the process are in this case the minimal specific energy of disintegration and the maximum drilling speed [31].

In practice, in some cases, spectrum changes are examined depending on the change of a given variable. For example, in the technical diagnostics of rotary machines, it is interesting to observe the change of spectrum of their vibration when increasing the revolutions (rpm). We talk about so-called spectrogram, i.e., spectrum dependence on time (or, in the example, on increasing revolutions).

Let's assume that we have measured a number of $s$ spectra corresponding to a time interval of $0,1,2, \ldots, s-1$.

This sequence of spectra represents the spectrogram as a highly integrative information source. In calculating its entropy as a potential information content, we must calculate the number $n_{|F| s}$ of possible spectrograms consisting of spectra $s$.

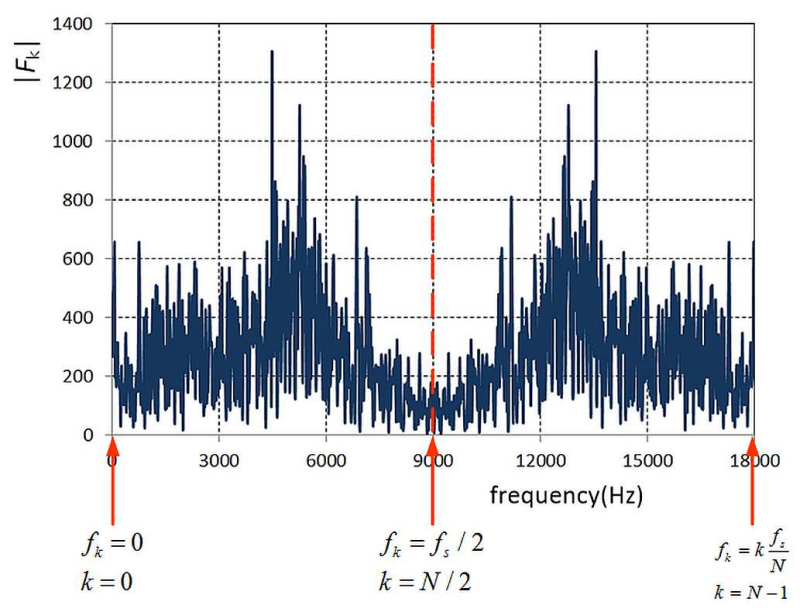

Figure 6 . The two-sided complex amplitude spectrum of an acoustic signal from the rock drilling process.

Based on previous considerations, starting from the combinatorial one, we can conclude that a spectrogram containing the spectra of signal records with a length of $N$ samples obtained by the $m$-bit $\mathrm{AD}$ converter represents variations of the $s$-class of $n_{|F|}$ elements with the repeating. Each of the spectra may occur at multiple time moments. If this equation is valid:

$$
n_{|F| s}=V_{s}^{\prime}\left(n_{|F|}\right)=n_{|F|}^{s} .
$$

Then the entropy $H_{|F| s}$ (bit) of measurement, based on the investigation of spectrogram of the sensor signal is given by the equation:

$$
\begin{aligned}
H_{|F| s}=\log _{2} n_{|F| s} & =\log _{2} n_{|F|}^{s} \\
= & s \log _{2} n_{|F|}=s \frac{N}{2} m
\end{aligned}
$$

If, for example, we used an $\mathrm{AD}$ converter with the width of $m=12$ (bit) to digitize the analog signal of the sensor and we would evaluate a spectrogram containing $s=10$ complex amplitude spectra, each of which was generated by analysing a signal recording with the length of $N=1024$ samples, then the entropy of such measurement would, according to (15), have the value of $H_{|F| s}=10 \frac{1024}{2} 12=61440$ (bit).

As an example of the spectrogram investigation, we can present the spectral analysis of an acoustic signal of the accompanying noise in the rock drilling process [32, 33]. The aim of the analysis is to obtain the information on the actual conditions of the rock disintegration by rotary drilling in terms of an optimal control of this process (see Figure 7) 34 37].

Thus, the increase of the entropy compared to the classical analog technique as well as in the time domain digital technique is significant in the case of the signal evaluation in the frequency domain. This is illustrated in Table 1.

To highlight the differences in measurement systems, the potential entropy values of the individual signal processing methods were recalculated to the decimal logarithm $\log _{10} H(\varphi)$. It is shown in Figure 8 


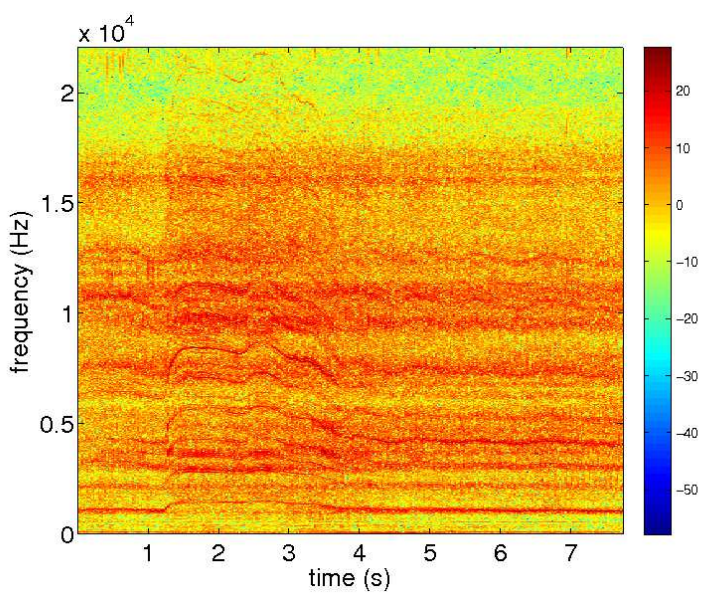

FiguRE 7. Spectrogram of acoustic signal as accompanying noise in rotary disintegration of granite.

\begin{tabular}{lc}
\hline Measuring system & Entropy \\
1 - Analogue system & $H_{a \delta}=5.67 \mathrm{~b}$ \\
2 - Digital system & $H_{\mathrm{DIG} m}=12 \mathrm{~b}$ \\
3 - Hybrid system & $H_{\mathrm{HYB}}=23.25 \mathrm{~b}$ \\
4 - Spectrum & $H_{f}=6144 \mathrm{~b}$ \\
5 - Spectrogram & $H_{|F| s}=61440 \mathrm{~b}$ \\
\hline
\end{tabular}

TABLE 1. Approximate entropy values for individual methods of evaluating the sensor signal.

\section{Summary AND CONCLUSIONS}

Table 1 shows the comparison of the individual measuring systems. Based on the entropy values of the sensor signal evaluation, it can be seen that the analog measurement system has the lowest information notice value. This is understandable because this system belongs to classical measurement systems, but is still used at the lowest procedural level of control. The digital measuring system is an extension of the analog system by a part, which ensures the conversion of the analog variable into a number in a suitable form and for subsequent processing. The hybrid system is an example of a measurement system in which the benefits of both systems are interconnected.

The signal processing of the sensor in terms of entropy in the frequency domain has a high information value. This is confirmed by the numerous uses in industrial practice and in various areas ranging from mining (e.g., processing of signals from geological survey wells) through the automotive industry (e.g., signal processing gerenerized by the car and its influence on the driver) to medicine (e.g., EKG cardiac signal processing, EEG brain). The successful implementation of the developed experimental measuring systems, and thus their practical applicability, is always decided by a deployment in a real environment.

It is necessary to say that the current industrial distributed control systems have an increasingly more complex and more extensive transmission and processing of data. Distributed control systems use a variety

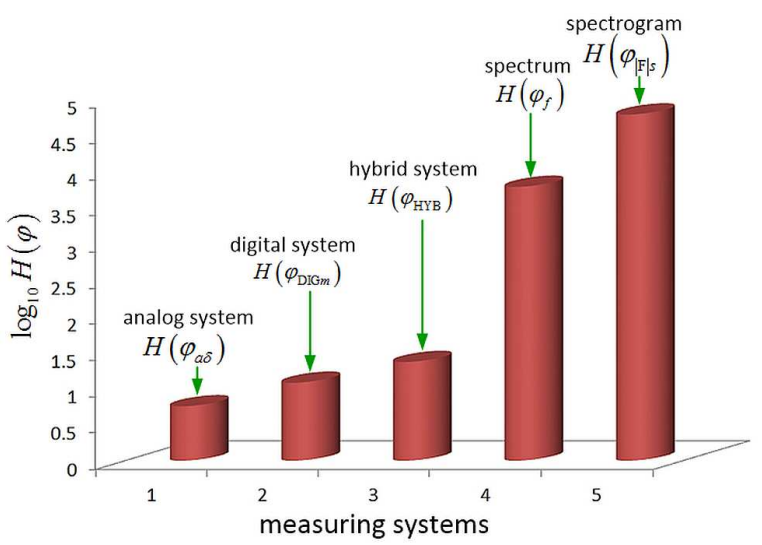

FiguRE 8. Potential entropy values of individual signal processing methods.

of communication buses. This means that at the lower levels of control, the necessary technical means are used with the digital processing of information from intelligent sensors, analyzers to PLC systems and workstations. At this lower level, the current state is characterized by the use of classic measurement systems along with intelligent or smart elements that are capable of cooperating through industrial communications networks.

The described problem is so serious when implementing new measurement systems or signal processing that it deserves an increased attention.

Verification of the correctness and effectiveness of the presented measuring systems was carried out in the framework of research activities and problem-oriented projects.

\section{ACKNOWLEDGEMENTS}

This work was supported by the Slovak Research and Development Agency under contract APVV-14-0892 and grants VEGA 1/0273/17 from the Slovak Grant Agency for Science.

\section{REFERENCES}

[1] C. Shannon. A mathematical theory of communication. Bell System Technical Journal 27(3):379-423 and 623-656, 1948. DOI:10.1109/9780470544242.ch1.

[2] C. Shannon, W. Weaver. The Mathematical Theory of Communication. The University of Illinois Press, Urbana IL, 1964. DOI:10.2307/3611062

[3] M. Belies, S. Guiasu. A quantitative-qualitative measure of information in cybernetic systems. In IEEE Trans. Inf. Theory IT-4, pp. 593-594. 1968. DOI:10.1109/tit.1968.1054185

[4] E. T. Jaynes. Information theory and statistical mechanics. Phys Rev 106(4):620-630, 1957. DOI:10.1103/physrev.108.171.

[5] A. Delgado-Bonal, J.Martín-Torres. Human vision is determined based on information theory. Scientific Reports 6(1), 2016. DOI:10.1038/srep36038 
[6] J. Shore, R. Iohnson. Axiomatic derivation of the principle of maximum entropy and the principle of minimum cross-entropy. IEEE Trans Inf Theory 26(1):26-37, 1980. DOI:10.1109/tit.1980.1056144

[7] A. Rényi. On measures of entropy and information. Proceedings of the Fourth Berkeley Symposium on Mathematical Statistics and Probability, Berkeley-Los Angeles pp. 547-561, 1961.

[8] M. Donald. On the relative entropy. Commun Math Phys 105:13-34, 1986. DOI:10.1007/bf01212339

[9] L. R. Nemzer. Shannon information entropy in the canonical genetic code. Journal of Theoretical Biology 415:158-170, 2017. DOI:10.1016/j.jtbi.2016.12.010.

[10] S. Yu, T.-Z. Huang, X. Liu, W. Chen. Information measures based on fractional calculus. Inf Process Lett 112(23):916-921, 2012. DOI:10.1016/j.ipl.2012.08.019.

[11] S. Yu, T.-Z. Huang. Exponential weighted entropy and exponential weighted mutual information. Neurocomputing 249:86-94, 2017. DOI:10.1016/j.neucom.2017.03.075

[12] K. Krechmer. Relational measurements and uncertainty. Measurement 93:36-40, 2016. DOI:10.1016/j.measurement.2016.06.058

[13] K. Krechmer. Relative measurement theory, the uniïnAzcation of experimental and theoretical measurements. Measurement 116:77-82, 2018. DOI:10.1016/j.measurement.2017.10.053

[14] N. Travers. Exponential bounds for convergence of entropy rate approximations in hidden markov models satisfying a path-mergeability condition. Stochastic Processes and their Applications 124(12):4149-4170, 2014. DOI:10.1016/j.spa.2014.07.011

[15] M. Thomas, J. Thomas. Elements of Information Theory. John Wiley and Sons, Inc. Print, 1991. DOI:10.1002/0471200611

[16] T. Schneider. Information theory primer with an appendix on logarithms. National Cancer Institute, 2007.

[17] P. Duhamel, M. Vetterli. Fast fourier transforms: A tutorial review and a state of the art. Signal Processing 19:259-299, 1990. DOI:10.1016/0165-1684(90)90158-u.

[18] A. V. Oppenheim, R. W. Schafer. Discrete-Time Signal Processing. Prentice-Hall, 1989.

[19] K. Nelson. A definition of the coupled-product for multivariate coupled-exponentials. Physica A: Statistical Mechanics and its Applications 422:187-192, 2015. DOI:10.1016/j.physa.2014.12.023.

[20] M. Frigo, S. G. Johnson. Fftw: An adaptive software architecture for the fft. In Proceedings of the International Conference on Acoustics, Speech, and Signal Processing, vol. 3, pp. 1381-1384. 1998. DOI:10.1109/icassp.1998.681704

[21] M. H. Hayes. Statistical Digital Signal Processing and Modeling. John Wiley and Sons, 1996.

[22] J. W. Cooley, J. W. Tukey. An algorithm for the machine computation of the complex fourier series. Mathematics of Computation 19:297-301, 1965. DOI: $10.2307 / 2003354$
[23] S. Shreedharan, C. Hegde, S. Sharma, H. Vardhan. Acoustic fingerprinting for rock identification during drilling. International Journal of Mining and Mineral Engineering 5(2):89-105, 2014. DOI:10.1504/ijmme.2014.060193

[24] H. Zheng, Y. Mingjun, S. Fuyu. A new method for measuring young's modulus by optical fiber sensor. In Proceedings of the 2012 Third International Conference on Mechanic Automation and Control Engineering, vol. 3 of MACE '12, pp. 1662-1664. IEEE Computer Society, 2012.

[25] J. Boroška, J. Krešák, P. Peterka. Estimation of quality for steel wire ropes according to their mechanical properties. Acta Montanistica Slovaca 1:37-42, 1997.

[26] E. Štroffek, I. Leššo. Acoustic method for measurement of young's modulus of steel wire ropes. Metalurgija 40(4):219-221, 2001.

[27] I. Leššo, J. Futó, F. Krepelka, et al. Control with acoustic method of disintegration of rocks by rotary drilling. Metalurgija 43(2):119-121, 2004.

[28] P. Peterka, P. Kačmáry, J. Krešák, et al. Prediction of fatigue fractures diffusion on the cableway haul rope. Engineering Failure Analysis 59:185-196, 2016. DOI:10.1016/j.engfailanal.2015.10.006

[29] I. Leššo, P. Flegner, et al. New principles of process control in geotechnics by acoustic methods. Metalurgija 46(3):165-168, 2007.

[30] G. Wittenberger, M. Cehlár, Z. Jurkasová. Deep hole drilling modern disintegration technologies in process of hdr technology. Acta Montanistica Slovaca 17(4):241-246, 2012.

[31] I. Leššo, P. Flegner, et al. Research of the possibility of application of vector quantisation method for effective process control of rocks disintegration by rotary drilling. Metalurgija 49(1):61-65, 2010.

[32] Masood, H. Vardhan, M. Aruna, B. R. Kumar. A critical review on estimation of rock properties using sound levels produced during rotary drilling. International Journal of Earth Sciences and Engineering 5(6):1809-1814, 2012.

[33] P. Flegner, J. Kačur, M. Durdán, et al. Measurement and processing of vibro-acoustic signal from the process of rock disintegration by rotary drilling. Journal of the International Measurement Confederation 56:178-193, 2014. DOI:10.1016/j.measurement.2014.06.025

[34] J. Jurko, A. Panda, M. Gajdoš. Study of changes under the machined surface and accompanying phenomena in the cutting zone during drilling of stainless steels with low carbon content. Metalurgija 50(2):113-117, 2011.

[35] J. Jurko, M. Dzupon, A. Panda, et al. Deformation of material under the machined surface in the manufacture of drilling holes in austenitic stainless steel. Chemicke listy 105(16):600-602, 2011.

[36] P. Flegner, J. Kačur, M. Durdán, et al. Significant damages of core diamond bits in the process of rocks drilling. Engineering Failure Analysis 59:354-365, 2016. DOI:10.1016/j.engfailanal.2015.10.016

[37] I. Leššo, P. Flegner, J. Futó, Z. Sabovaá. Utilization of signal spaces for improvement of efficiency of metallurgical process. Metalurgija 53(1):75-77, 2014. 